\title{
VISIÓN DE LA REALIDAD Y LOS FACTORES RELACIONADOS CON LA TOMA DE CONCIENCIA DE JÓVENES EN VIEQUES ${ }^{1}$
}

\author{
Erika Migliza Candelaria Arce 2 \\ Waleska Janice Rivera Oquendo ${ }^{3}$
}

\begin{abstract}
"La participación de los jóvenes en organizaciones y movimientos de base, particularmente en los espacios: ecologistas, de mujeres, comunitarios, culturales, es muy alta y esperanzadora. Los que hemos trabajado en estos espacios sabemos el tipo de aporte que los jóvenes están haciendo alli: creando, innovando en formas de organización e interacción, gestando ciudadanía y capital social, sirviendo de articuladores entre la tradición -y las nuevas tecnologias. Es importante aprender de estas experiencias y utilizarlas en el proceso de diseñar políticas sociales y hacer cambios en la estructura y funciones del Estado. Por otro lado vale la pena destacar los aportes en el campo cultural que hacen los jóvenes... En fin, tenemos que aprender a mirar y a escuchar las voces de la juventud y comprometernos a ser realidad el pilar fundamental de la democracia en la que decimos creer". ${ }^{4}$
\end{abstract}

\footnotetext{
${ }^{1}$ Artículo basado en la tesis de maestría en la Escuela Graduada de Trabajo Social, a mayo del 2001.

${ }^{2}$ Trabajadora Social, Programa Trabajo Social Obrero.

${ }^{3}$ Estudiante doctoral, Universidad Autónoma de Nuevo León, Monterrey, México.

${ }^{4}$ Marcia Rivera, Representante del Consejo Latinoamericano de las Ciencias Sociales (CLACSO) ante la VIII Conferencia Iberoamericana de Ministros de Juventud en Buenos Aires, Argentina, señala.
} 


\section{Definición del Problema y Justificación}

La actividad civil existente en el Municipio de Vieques constituye terreno fértil para la exploración de los factores relacionados a la participación ciudadana. Se encuentra que uno de los sectores involucrados en el esfuerzo por alcanzar la paz en Vieques es el compuesto por los jóvenes, quienes como grupo social en el transcurrir del tiempo, se han caracterizado por una participación activa en las luchas comunitarias. No obstante, poco se ha estudiado acerca de los factores asociados a su visión de realidad y decisión de participación, como grupo, en las situaciones comunitarias. Por tal razón el presente estudio se propuso explorar y describir los factores que inciden en la visión de la realidad y en el proceso de tomar conciencia en la población juvenil viequense entre las edades de 14-24 años.

\section{Revisión de Literatura y Marco Conceptual}

Estudios sobre la juventud puertorriqueña revelan la existencia de una preocupación juvenil por lo que ocurre en la sociedad; pero los jóvenes muestran pesimismo sobre el futuro del país y el resto del mundo (Mac Cann Erickson, 1995; Rodríguez y Collazo, 1995), manifestando que se sienten sin liderazgo y hasta cierto punto marginados y confundidos (Mac Cann Erickson, 1995). De lo anterior se podría interpretar que el deseo y la disposición están presentes entre los jóvenes pero los canales adecuados escasean, promoviendo el desarrollo de una cosmovisión de minusvalía ante los problemas sociales que enfrentan.

Se trata de la cosmovisión del mundo o formación ideológica $\mathrm{del} /$ la joven la que constituye su concepción política, económica, social, ética y religiosa o sea su visión de la realidad. Se concibe la realidad como aquello de lo que se puede enunciar algo; lo que ocurre verdaderamente, lo efectivo o lo que tiene valor práctico, lo evidente (Enciclopedia Larrouse, 1995). Para Francia y Salmerón (1993) la realidad social es el conjunto de fenómenos, hechos y situaciones que se dan como consecuencias de la interacción humana. Esta tiene una apariencia externa, de la que se puede extraer información y una esencia, a la que se puede llegar mediante un proceso ordenado y reflexivo. 
Cuando se estudia la realidad se deben determinar los condicionantes pasados, presentes y futuros así como la historia de los fenómenos. El conocimiento de la realidad parte de dos perspectivas: los criterios metodológicos de quienes analizan y las intenciones y sentimientos de los sujetos tal y como son expresados por ellos mismos.

Analizar la realidad desde el ámbito socio comunitario puede hacerse bajo un criterio poblacional, considerando en este caso el análisis del sector juvenil viequense. Dentro de este ámbito existen tres dimensiones identificadas por Rubio y Varas (1999) la infraestructura, la estructura y la supraestructura. La dimensión de la infraestructura recoge información sobre los recursos económicos de los jóvenes, características demográficas relativas a la distribución de edades, sexo, estado civil, nivel de estudios, entre otros. En la dimensión estructural se analizaran las redes de apoyo sociales, asociaciones y las relaciones con familiares y otros sujetos. En cambio, en la dimensión de la supraestructura se consideran tanto la percepción social sobre los jóvenes como su auto percepción, sus valores y sus creencias. En cuanto al ámbito psico-social se estudia la estructura de las relaciones entre individuos que llevan a constituir organizaciones y grupos. Los elementos psico-sociales son los roles, las tareas, la comunicación, la afectividad, el status, las características individuales, las normas, entre otros. Para finalizar se encuentra el ámbito psicológico o microsistema. Estos son los elementos que constituyen en su interrelación la estructura de personalidad de cada individuo. Estos elementos pertenecen a tres dimensiones: biológica, psicológica y social. Se trata de considerar a la persona desde un enfoque bio-psico-social. Al ámbito bio-psico-social algunos teóricos le añaden el aspecto espiritual (Fowler,1981). Se concibe el mismo como la relación del ser humano con elementos trascendentes como pueden ser valores, principios y filosofías de vida.

La cosmovisión del / la joven, parte de los elementos antes mencionados y guarda una relación dialéctica con el proceso de toma de conciencia. Marx (1859), indica que la conciencia se trata de un producto social que implica la expansión de las actividades de las personas, las relaciones sociales y la producción de la vida material en el desarrollo de la persona. Por otro lado tomar conciencia se trata del proceso en el que el ser humano conoce y modifica su realidad; esta nueva realidad entra en una nueva relación con el ser humano y le exige compromisos de una visión profunda de sí mismo 
y a la vez lo capacita para una visión de la realidad más profunda (Vela, 1990). Ander Egg (1995) señala que tomar conciencia es más que la experiencia reflexiva, es saber explicar, qué se sabe, qué se conoce y cómo se conoce.

El proceso de tomar conciencia se genera en una tensión entre la conciencia del presente histórico y la conciencia de una esperanza creadora, de una historia nueva (Vela, 1990). Este proceso se trata de uno en espiral compuesto por tres momentos fundamentales, en primer lugar la identificación de fenómenos o la realidad a simple vista. Como segundo momento se encuentra la codificación, el mismo hace referencia a las significaciones de los fenómenos observados. En tercer lugar se da la descodificación, donde partiendo de la reflexión personal y el diálogo intergrupal se definen las relaciones, problemática y posibilidad de creatividad para la solución de los problemas.

Para Freire, tomar conciencia es un acto del conocimiento que implica un develamiento de la realidad, en la que la persona se va adentrando poco a poco en la esencia misma de los hechos que tenga frente a sí. Esta conciencia del mundo conlleva una opción ideológica acompañada de un proceso psicológico individual y social que parte de la realidad misma (Freire, 1974).

Es necesario despertar a la posibilidad de cambiar la realidad. Esto es posible mediante el desarrollo de un pensamiento crítico de las circunstancias cotidianas del ser humano. Pepín Leistyna (1999) señala, que solo mediante el pensamiento crítico se alcanzará una sociedad civil responsable con una fuerte participación política para la justicia social. Pero el pensamiento crítico por si solo no es la conciencia.

El pensamiento crítico es:

"La capacidad para la dialogicidad, que hace que el pensamiento se reconozca como parte de un diálogo, que reconozca la multiplicidad de lógicas o interpretaciones y que se vea obligada a fundir adecuadamente la suya y a integrarla" (Villarini, 1987).

Una vez alcanzada la capacidad del pensamiento crítico será posible el desarrollo de una conciencia crítica de la realidad a la que se enfrenta la persona. Dice Freire (1974) que mientras más concientizadas, más capacitadas están las personas para el compro- 
miso de transformación que asumen. Freire (1974) recalca que este compromiso con la transformación de las circunstancias opresoras debe ser permanente. Indica que a partir de la denuncia de una estructura deshumanizante sin un verdadero compromiso con la realidad se burocratiza el esfuerzo.

El estar concientizados críticamente implica el tomar posición ante la realidad. En otras palabras implica el participar en ella. La participación es definida por Ander Egg (1995) como: la influencia sobre el proceso de toma de decisiones a todos los niveles de la actividad social y las instituciones sociales. Esta acción se relaciona entonces con el acceso al poder. Hopehayn (1988) señala como iniciativas para la participación:

1. Ganar control sobre la propia situación y proyecto de vida mediante la intervención en decisiones que afectan el entorno vital en dicha situación y proyecto que desenvuelven.

2. Accesar a mejores y mayores bienes y/o servicios en la sociedad.

3. Integrarse a procesos de desarrollo en las cuáles los sectores excluidos se constituyen en chivos expiatorios.

4. Aumentar el grado de autoestima gregaria mediante un mayor reconocimiento por parte de los derechos, las necesidades y las capacidades propias.

El mismo autor señala que la participación tiene sentido si redunda en humanización. Si se adquiere mediante ésta mayor presencia en la asignación social de lo socialmente producido, si se remueven obstáculos socioeconómicos que sean estructurales o institucionales que impiden el desarrollo de la persona. Como participación efectiva se identifican la asociación, la delegación de poder y el control por los ciudadanos.

Partiendo del señalamiento anterior se enmarcará el proceso de concienciación en la visión de la realidad de la juventud en Vieques. En el 1940 interviene en la historia de Vieques, la Marina de Guerra del Ejército de los Estados Unidos. Con su llegada a la Isla se impuso la re localización de las actividades civiles en una franja equivalente a una tercera parte del área total de Vieques. Se indica en el Plan de Ordenamiento territorial para Vieques (2000) que 
durante el Siglo XX la Marina constituyó el principal agente de cambio en material físico- espacial- político- ideológico y socioeconómico del territorio. Esta presencia militar coincidió y precipitó la reducción y colapso de las industrias del azúcar y el ganado existente en la municipalidad.

La Isla de Vieques cuenta con una población de 9,637 habitantes según las proyecciones del 2000 , de éstos un $32.6 \%$ son jóvenes y niños de 19 años o menos. Vieques posee el mayor porcentaje de pobreza en Puerto Rico con un 70.2\%. Su tasa de desempleo para el 1999 según el Departamento del Trabajo fue de 13.1\%, cifra que sobrepasa la del resto de la isla, siendo los jóvenes el sector poblacional más afectado por el desempleo.

A nivel educativo se identifica en Vieques un serio problema de deserción escolar, que llega al 2.6\% en comparación al 1.5\% registrado en la misma fecha (1995) en el resto de Puerto Rico. Al momento de la investigación, Vieques cuenta con 12 escuelas y un institución post secundaria (Manpower Business Training Institute- MBTI).

En cuanto el nivel de salud de la Isla Nena, cabe señalar que según las estadísticas de mortalidad de 1995 Vieques tiene $40 \%$ más casos de mortalidad que el resto de Puerto Rico (Plan de Ordenamiento Territorial, 2000). Se encontró que las tres razones principales de muerte en Vieques son: los accidentes, las enfermedades del corazón y el cáncer. El último informe del Registro de Control de Cáncer de 1991, señaló que la tasa de mortandad en Vieques fue de 200.7\% seguido por San Juan con una tasa de mortandad de cáncer de 163.7\% (Massol, 2000; Plan de Ordenamiento Territorial, 2000).

A partir del accidente en prácticas de la Marina de Guerra de Estados Unidos que le costó la vida al guardia de seguridad David Sanes, se ha generado un proceso de discusión donde se ha puesto, una vez más, ante el tapete público la temática en torno a la presencia de la Marina de Guerra y su impacto en la vida de los residentes de Vieques. Las actividades de la Marina Estadounidense han tenido un efecto perjudicial y detrimental sobre la calidad de vida de sus residentes (Informe de la Comisión Especial de Vieques) y que los viequenses han estado denunciando durante los pasados 60 años.

Hoy día la juventud ha asumido un importante papel para cumplir con este compromiso realizado. Es necesario destacar el acto heroico de los hermanos Zenón y otros jóvenes, quienes luego de los primeros arrestos se han mantenido realizando actividades de protestas y manifestaciones a favor de la paz de Vieques. Al respec- 
to del espíritu juvenil viequense, el licenciado Fermín Arraiza señala que: "Uno se tira a la calle a hablar con el pueblo, y se da cuenta que ya no es la batalla de los legendarios pescadores, la lucha es de todo el pueblo y la juventud tiene una presencia fuerte, están dispuestos a luchar por respetar sus derechos civiles"

\section{Estudio}

\section{Diseño Metodológico}

El presente estudio tuvo como finalidad el conocer la visión de la realidad y el proceso de tomar conciencia de los jóvenes viequenses entre las edades de 14 -24 años, desde un acercamiento exploratorio descriptivo. Se utilizó como instrumento de recopilación de datos un cuestionario, donde se exploraron los aspectos de visión de la realidad, reconocimiento de las fuerzas que actúan sobre esta realidad y las respuestas que como jóvenes les han dado a la problemática existente.

\section{Hallazgos}

El instrumento de investigación fue administrado entre los meses de mayo a junio del 2000 a 128 jóvenes viequenses entre las edades de 14-24 años.

El sujeto típico de este estudio pertenece al género femenino $(50.8 \%)$ entre las edades de 14 a 17 años, estudiante $(68.8 \%)$, soltera, perteneciente al credo metodista cuyos ingresos familiares procedían de un empleo oficial.

Un $7 \%$ de los jóvenes estudian y trabajan. Mientras un $8.6 \%$ de los jóvenes señalaron estar desempleados. Se encontró que aproximadamente el $50 \%$ de los encuestados indicaron que la mayor dificultad para conseguir empleo es la poca disponibilidad de estos en Vieques. Segunda dificultad señalada para conseguir empleo, fue los bajos salarios de los trabajos disponibles (13.3\%), mientras que la tercera dificultad para conseguir empleo fue la falta de experiencia $(9.4 \%)$.

En el Censo de 1990 se encontró que la tasa de desempleo en Puerto Rico era de $20.4 \%$, mientras que en Vieques la tasa alcanzaba un $26.3 \%$. En Vieques se agrava la situación por el aislamiento 
geográfico, por la falta de agencias que brinden servicio a la población y la carencia de iniciativas alternas para el desarrollo económico. Los viequenses que trabajan en la Isla Municipio tienen trabajos de mantenimiento, guardias de seguridad o comercio, donde le pagan el mínimo por hora. En el estudio La Selección Vocacional de los Estudiantes de la Escuela Superior de Vieques Comparada con las Oportunidades de Empleos Existentes en Vieques (1968) se encontró que no existía oportunidad de empleos para la gran mayoría de estudiantes graduados de la escuela superior, situación que no ha variado desde entonces.

\section{Aspiraciones}

ASPIRACIÓN ACADÉMICA

\begin{tabular}{lcc}
\hline Grado & Frecuencia & Por ciento \\
\hline Bachillerato & 44 & 34.4 \\
Doctorado & 20 & 15.6 \\
Maestría & 18 & 14.1 \\
Curso técnico/ Vocacional & 17 & 13.3 \\
Cuarto año & 14 & 10.9 \\
No interesa continuar estudios & 3 & 2.3 \\
Ninguna & 12 & 9.4 \\
& & $100 \%$ \\
\hline Total & $\mathbf{1 2 8}$ & \\
\hline
\end{tabular}

El 77.3\% de los jóvenes aspiraban obtener algún grado mayor al de cuarto año de escuela superior, tal vez esperando que con una mayor educación puedan encontrar mejores empleos.

De acuerdo con la información provista por los jóvenes, el factor económico es la limitación principal (19.5\%) para estudiar, seguido de la transportación (14.8\%). Cabe destacar que, por ejemplo, un joven universitario que trabaje para poder pagar sus estudios y gastos relacionados como hospedaje, comida y otros tendrá dificultad para poder beneficiarse de los servicios de transportación pública ya que los horarios existentes son muy limitados. 
La siguiente tabla ilustra que en 34 jóvenes (36.6\%) su aspiración ocupacional está relacionada con el área de comercio. En ésta área se encontraron los trabajos de finanzas, ventas, bienes raíces y tener negocio propio. Cabe destacar que algunos trabajos relacionados con el comercio son de servicios de dependientes como atender alguna tienda. Solamente 2 jóvenes desearon tener su propio negocio en Vieques, mientras 17 participantes (13.3\%) indicaron que su meta ocupacional es trabajar en el gobierno. Por otro lado, ocho de los jóvenes (6.3\%) entrevistados aspiran ser pescadores. Algunos jóvenes que señalaron otras ocupaciones mencionaron profesiones como ingeniería, carreras relacionadas con la medicina y ciencias naturales, entre otras. No obstante, para ejercer la mayoría de estas profesiones y ocupaciones tienen que emigrar fuera de Vieques o del país. Esta observación es confirmada al solo el dieciocho por ciento tener la esperanza de obtener el empleo deseado en Vieques.

META OCUPACIONAL

\begin{tabular}{lcc}
\hline Sector de empleo & Frecuencia & Por ciento \\
\hline Comercio & 40 & 31.3 \\
Gobierno & 21 & 16.4 \\
Pesca & 9 & 7.0 \\
Construcción & 6 & 4.7 \\
Transportación & 4 & 3.1 \\
Fábrica & 3 & 2.3 \\
Agricultura & 2 & 1.6 \\
Otros (No especificó) & 27 & 21.0 \\
Indecisos & 16 & 12.5 \\
\hline Total & 128 & $\mathbf{1 0 0 \%}$ \\
\hline
\end{tabular}




\section{Visión Sobre la Vida}

\section{METAS DE VIDA: PERSONALES}

\begin{tabular}{lcc}
\hline Meta & Frecuencia & Por ciento \\
\hline Sentir que ha logrado mucho & 41 & 32.0 \\
Vida cómoda & 37 & 28.9 \\
Vida activa y excitante & 22 & 17.2 \\
Vivir la vida sabiamente & 18 & 14.1 \\
Alcanzar felicidad & 17 & 13.3 \\
Gozar de las bellezas & 15 & 11.7 \\
Gozar de los placeres & 8 & 6.3 \\
Lograr respeto de la gente & 5 & 3.9 \\
Tener libertad de acción & 4 & 3.1 \\
\hline
\end{tabular}

${ }^{1}$ Para esta premisa se podía seleccionar más de una alternativa.

De acuerdo con los datos recopilados en el estudio, 41 jóvenes $(32.0 \%)$ tienen como meta personal de vida el sentir que han logrado mucho en la vida. Mientras que 37 de estos (28.9\%) optan por tener una vida cómoda y otros ocho $(6.3 \%)$ señalaron como meta personal el gozar de los placeres de la vida. Por otro lado, 5 jóvenes (3.9\%) indicaron como meta personal de vida el lograr el respeto de la gente y unos $4(3.1 \%)$ desean alcanzar la libertad de acción.

Las metas de vida identificadas por los jóvenes responden al momento en que se encuentran los mismos. Por ejemplo, 32 por ciento de los entrevistados señaló como meta personal el sentir que ha logrado mucho en la vida. Además el deseo de tener una vida activa y excitante responde a la etapa de vida en que se encuentran estos jóvenes, donde la aventura y lo innovador es una de las motivaciones principales para el ser humano. Sin embargo, también establecen como meta prioritaria el vivir la vida sabiamente. Esto podría implicar el desarrollo de un pensamiento crítico . 
METAS SOCIALES DE VIDA

Meta

Lograr el bienestar de su familia

Luchar por la paz en el mundo

Alcanzar un amor de pareja verdadero y profundo

Tener hijos

Lograr igualdad de oportunidades para todos

Tranquilidad espiritual

Salvar el alma

Tener verdaderos amigos

Sentir que vale mucho
Frecuencia* Por ciento

35.9

28.9

24.2

14.8

13.3

17

8.6

11

8.6

10

7.8

$5 \quad 3.9$

* Para esta premisa se podía seleccionar más de una alternativa.

Según los datos encontrados, 46 jóvenes $(35.9 \%)$ tienen como metas sociales de vida el lograr el bienestar de su familia. Mientras unos 37 jóvenes (28.9\%) optaron como meta de vida el luchar por la paz en el mundo. Otros 31 jóvenes (24.2\%) tienen como meta alcanzar un amor de pareja verdadero y profundo, además de un $14.8 \%$ que desean tener hijos.

Las metas de lograr el bienestar de la familia junto con las metas de alcanzar un amor de pareja verdadero y profundo, y con el tener hijos responden al interés de tener un hogar estable y seguro para su propio desarrollo y el de su familia. A estas metas se le añade el interés por luchar por la paz en el mundo, lo cual está muy relacionado con el deseo de estos jóvenes de que haya paz en Vieques .

\section{Modo de ver la vida}

\section{Experiencias significativas}

En las ciencias sociales se ha comprobado que la visión es un estilo de mirar al mundo partiendo de las experiencias individuales de cada sujeto. Las experiencias más significativas llegan s influenciar en la manera en que percibe el mundo el joven. En el estudio realizado se pudo corroborar que las experiencias familiares (63.3\%) fueron las más significativas. Según Berger y Luckman (1976) las experiencias son necesarias para que el sujeto llegue a integrarse en la sociedad y se relacione tanto con personas como 
con instituciones. Las fuentes principales de información se tratan de los medios de comunicación masiva. No obstante, para 73 de los participantes (57\%) las experiencias con la lucha por sacar a la Marina de Vieques han influido mucho en la vida de estos. El sentirse útil y parte de una causa son experiencias que fortalecen la autoestima de una persona. Esto es mayor cuando la persona se encuentra en la etapa de ser reconocido por los demás y sentirse parte de un grupo.

\section{EXPERIENCIAS SIGNIFICATIVAS}

\begin{tabular}{|c|c|c|c|c|c|c|c|c|c|c|}
\hline \multirow[t]{2}{*}{ Tipo de experiencia } & \multicolumn{2}{|c|}{ Mucho } & \multicolumn{2}{|c|}{ Poco } & \multicolumn{2}{|c|}{ Nada } & \multicolumn{2}{|c|}{ No contestó } & \multicolumn{2}{|c|}{ Total } \\
\hline & $F$ & $\%$ & $\mathbf{F}$ & $\%$ & $\mathbf{F}$ & $\%$ & $\mathbf{F}$ & $\%$ & $\mathbf{F}$ & $\%$ \\
\hline Familiar & 81 & 63.3 & 27 & 21.1 & 8 & 6.3 & 12 & 9.4 & 128 & 100 \\
\hline Eclesial & 35 & 27.3 & 69 & 53.9 & 13 & 10.2 & 11 & 8.6 & 128 & 100 \\
\hline Amigos & 72 & 56.3 & 31 & 24.2 & 10 & 7.8 & 15 & 11.7 & 128 & 100 \\
\hline \multicolumn{11}{|l|}{ La lucha por sacar a } \\
\hline la Marina de Vieques & 73 & 57.0 & 22 & 17.2 & 171 & 13.3 & & 12.5 & 128 & 100 \\
\hline Estudiantil & 83 & 64.8 & 26 & 20.3 & 5 & 3.9 & & 10.9 & 128 & 100 \\
\hline Con el barrio & 46 & 35.9 & 40 & 31.3 & 30 & 23.4 & & 29.4 & 128 & 100 \\
\hline
\end{tabular}

\section{PERSONAS INFLUYENTES*}

\begin{tabular}{|c|c|c|c|c|c|c|c|c|}
\hline \multirow[t]{2}{*}{ Personas } & \multicolumn{2}{|c|}{ Mucho } & \multicolumn{2}{|c|}{ Poco } & Nada & No contestó & \multicolumn{2}{|c|}{ Total } \\
\hline & $\mathbf{F}$ & $\%$ & $F$ & $\%$ & F $\%$ & F \% & $F$ & $\%$ \\
\hline Padre & 745 & 57.8 & 27 & 21.1 & 1814.1 & 97.0 & 128 & 100 \\
\hline Madre & 947 & 73.4 & 14 & 10.9 & 97.0 & 118.6 & 128 & 100 \\
\hline Tío (a) & 34 & 26.6 & 60 & 46.9 & 1914.8 & 1511.7 & & 100 \\
\hline Abuelo (a) & 513 & 39.8 & 45 & 35.2 & 2116.4 & 118.6 & & 100 \\
\hline Religioso (a) & 30 & 23.4 & 43 & 33.6 & 3829.7 & 1713.3 & & 100 \\
\hline Dirigente de equipo & 15 & 11.7 & 36 & 28.1 & 6248.4 & 1511.7 & & 100 \\
\hline Líder comunitario & $11 \varepsilon$ & 8.6 & 24 & 18.8 & 7457.8 & 1914.8 & 128 & 100 \\
\hline Líder político & 97 & 7.0 & 21 & 16.4 & 8163.3 & 1713.3 & 128 & 100 \\
\hline Vecino (a) & 14 & 10.9 & 45 & 35.2 & 5240.6 & 1713.3 & 128 & 100 \\
\hline Amigo (a) & 73 & 57.0 & 30 & 23.4 & 118.6 & 1410.9 & 128 & 100 \\
\hline Hermano (a) & 65 & 50.8 & 26 & 20.3 & 1511.7 & 2217.2 & 128 & 100 \\
\hline Maestro (a) & 42 & 32.8 & 46 & 35.9 & $17 \quad 13.3$ & 2318.0 & 128 & 100 \\
\hline
\end{tabular}

*Hubo 29 sujetos que señalaron otra persona significativa pero no especificaron. 
La tabla anterior indica que 94 de los jóvenes (73.4\%) señalaron a la madre como la persona que más a influenciado en la opinión sobre la vida. La figura paterna $(57.8 \%)$ fue señalada en un lugar significativo. Este por ciento es casi igual a la influencia de los amigos $(57.0 \%)$. Este hallazgo se relaciona con las etapas de identidad vs. ego e identidad vs. aislamiento de Erikson (1968) porque para los jóvenes la relación con sus pares $\mathrm{u}$ familiares son muy significativas en el proceso de tomar decisiones, establecer una escala de valores, desarrollar la personalidad y de sus relaciones interpersonales.

\section{Visión sobre aspectos políticos}

En general la visión sobre los políticos es una poco beneficiosa para éstos ya que la gran mayoría de los participantes consideran que los políticos no realizan su trabajo como deberían. Además descartaron la poca participación que se les brinda para participar en la política. Con esto se podría interpretar que los jóvenes consideran que los políticos no tienen formas nuevas y creativas para trabajar con la problemática tanto de Vieques como de Puerto Rico. Los participantes opinaron que los políticos no cumplen con sus obligaciones porque tan solo se fijan en los intereses individuales donde puedan obtener la mayor ganancia mientras estén en el poder. Esta opinión coincide con la poca influencia que tienen los políticos en la vida de los jóvenes.

\section{VISIÓN SOBRE LOS POLÍTICOS}

\begin{tabular}{|c|c|c|c|c|c|c|c|c|}
\hline \multirow[t]{2}{*}{ Visión* } & \multicolumn{2}{|c|}{ Mucho } & \multicolumn{2}{|c|}{ Poco } & \multicolumn{2}{|c|}{ Nada } & \multicolumn{2}{|c|}{ No sé } \\
\hline & $\mathbf{F}$ & $\%$ & $\mathbf{F}$ & $\%$ & $\mathbf{F}$ & $\%$ & $\mathbf{F}$ & $\%$ \\
\hline Cumplen con sus obligaciones & 7 & 5.5 & 53 & 41.4 & 42 & 32.8 & 18 & 14.1 \\
\hline $\begin{array}{l}\text { Brindan espacio para la } \\
\text { participación de los jóvenes }\end{array}$ & 11 & 8.6 & 57 & 44.5 & 42 & 32.8 & 7 & 5.5 \\
\hline Son honrados & 8 & 6.3 & 39 & 30.5 & 52 & 40.6 & 18 & 14.1 \\
\hline $\begin{array}{l}\text { Están interesados en resolver } \\
\text { los problemas de Vieques }\end{array}$ & 21 & 16.4 & 42 & 32.8 & 45 & 35.2 & 13 & 10.3 \\
\hline Atienden a los que lo necesitan & 14 & 10.9 & 56 & 43.8 & 39 & 30.5 & 12 & 9.4 \\
\hline $\begin{array}{l}\text { Tienen preferencias sobre los } \\
\text { ricos }\end{array}$ & 54 & 42.2 & 13 & 10.2 & 15 & 11.7 & 35 & 27.3 \\
\hline $\begin{array}{l}\text { Sirven sólo a los que pertene- } \\
\text { cen a su mismo partido }\end{array}$ & 69 & 53.9 & 15 & 11.7 & 9 & 7.0 & 24 & 18.8 \\
\hline Son corruptos & 73 & 57.0 & 12 & 9.4 & 7 & 5.5 & 25 & 19.5 \\
\hline
\end{tabular}

* Para esta premisa se podía seleccionar más de una alternativa. 


\section{Problemas identificados por los jóvenes}

La gran mayoría de los jóvenes $(63.3 \%)$ han expresado que el desempleo es el problema principal en Vieques. Este hallazgo es congruente con el Censo de 1990 donde la tasa de desempleo en Puerto Rico era de 20.4 por ciento mientras en Vieques la tasa figuraba en el 26.3 por ciento. Una de las razones por las cuales entienden que el desempleo es el primordial problema es porque en el municipio hay poca disponibilidad de empleos. Este problema lo agrava el aislamiento geográfico, la falta de agencias que brinden servicios a la población y el que gran parte de la economía no está en manos de los mismos viequenses.

En cuanto a los próximos seis problemas identificados por los participantes se observa que todos están relacionados al área de salud, respectivamente: la drogadicción (51.6\%), el cáncer (37.5\%), el embarazo en adolescentes (26.6\%), la contaminación ambiental (24.4\%), los servicios de salud (14.8\%) y el alcoholismo $(14.1 \%)$.

El 13.3 por ciento (17) de los jóvenes señalaron que la recreación es un problema que les afecta directamente. No obstante las principales actividades realizadas en su tiempo libre eran: ir a la playa, practicar algún deporte y ver televisión. Cabe señalar que en el proceso de administrar el instrumento algunos jóvenes, informaron que una de las actividades que más disfrutaban era el patinaje, pero estaban limitados a hacerlo por una serie de leyes municipales.

\section{PROBLEMAS IDENTIFICADOS POR LOS JÓVENES}

\begin{tabular}{lcc}
\hline Problema & Frecuencia $^{1}$ & Por ciento \\
\hline Desempleo & 81 & 63.3 \\
Drogadicción & 66 & 51.6 \\
Cáncer & 48 & 37.5 \\
Embarazo en adolescentes & 34 & 26.2 \\
Contaminación ambiental & 31 & 24.2 \\
Servicios de salud & 19 & 14.8 \\
Alcoholismo & 18 & 14.1 \\
Recreación & 17 & 13.3 \\
Vivienda & 14 & 10.9 \\
Deserción & 14 & 10.9 \\
Criminalidad & 5 & 3.9 \\
Transportación & 4 & 3.1 \\
Enseñanza inadecuada & 2 & 1.6 \\
No especificado & 15 & 11.7
\end{tabular}

${ }^{1}$ Para esta premisa se podía seleccionar más de una alternativa. 


\section{Marina}

\section{NIVEL DE RELACIÓN DE ALGUNOS PROBLEMAS CON LA PRESENCIA DE LA MARINA}

\begin{tabular}{lcc} 
Problema & Frecuencia $^{1}$ & Por Ciento \\
\hline Falta de vivienda & 52 & 40.6 \\
Embarazo en adolescentes & 45 & 35.2 \\
Alcoholismo y drogadicción & 42 & 32.8 \\
Criminalidad & 23 & 18.0 \\
Violencia doméstica & 20 & 15.6 \\
\hline
\end{tabular}

A los jóvenes participantes se le presentó una lista de problemas para que estimaran el nivel de relación de estos con la Marina. De los diferentes problemas presentados, los que mayor relación para los jóvenes participantes guardan con la presencia militar son: la falta de vivienda (40.6\%), el embarazo en adolescentes $(35.2 \%)$ y el consumo de drogas y alcohol (32.8\%). De estos el consumo de drogas y alcohol y el embarazo en adolescentes se encuentran entre los primeros cuatro problemas existentes en la isla municipio de Vieques identificados por los participantes y mencionados en el Informe de la Comisión Especial de Vieques (1999).

\section{CAMBIO DE OPINIÓN SOBRE LA PRESENCIA DE LA MARINA}

\begin{tabular}{lcc}
\hline Cambio & Frecuencia & Por Ciento \\
\hline Sí & 67 & 52.3 \\
No & 61 & 47.6 \\
\hline Total & 128 & 100 \\
\hline
\end{tabular}

Por la especial atención prestada a la problemática de Vieques con la presencia de la Marina en los últimos años se les preguntó a las personas participantes si su opinión respecto a la presencia de la Marina en los últimos dos años había cambiado. Al respecto se observa que el 52.3 por ciento reconoció haber tenido algún cambio en su opinión.

\footnotetext{
${ }^{1}$ Para esta premisa se podía seleccionar más de una alternativa.
} 
Este cambio de opinión puede ser identificado como un cambio o transformación en la visión de la realidad de los jóvenes viequenses encuestados. Shipani y Freire (1992) indican que esta transformación es producto de un reordenamiento básico de la realidad, y una evaluación de nuevas posibilidades dentro de la realidad. Esta se trata de una segunda etapa de desarrollo de la concienciación, que en el caso de los jóvenes bajo estudio puede significar el desarrollo de alguna acción a favor de la paz en Vieques.

A los jóvenes se le solicitó que identificaran como había sido este cambio de opinión. El 40.6 por ciento indicó haber cambiado su opinión en contra de la presencia de la Marina en el Municipio. Las razones principales señaladas por los jóvenes para este cambio fueron sus vivencias en Vieques (20.3\%), la muerte de David Sanes (18.0 $\%)$ y la información referente al impacto de la presencia de la Marina (11.7\%). Estas experiencias del sujeto van estructurando su visión referente a la realidad. Además, cabe destacar que las vivencias del cotidiano vivir y las discusiones son fundamentales en el desarrollo del paradigma de la realidad que tienen estos jóvenes puertorriqueños.

\section{EFECTOS DE LA PRESENCIA DE LA MARINA EN LOS VIEQUENSES}

\begin{tabular}{lcc}
\hline Asistencia & Frecuencia $^{{ }^{1}}$ & Por Ciento \\
\hline Peores condiciones de salud & 83 & 64.8 \\
Expropiaciones de terrenos & 71 & 55.5 \\
Desarrollo económico y social deficiente & 68 & 53.1 \\
Solución de varios problemas & 15 & 11.7 \\
Aumento de empleos & 14 & 10.9 \\
Fortalecimiento de la economía & 1 & 0.8 \\
Otras no especificadas & 6 & 4.7 \\
\hline
\end{tabular}

En cuanto a los efectos de la presencia de la Marina en Vieques, son los efectos negativos los que con mayor frecuencia señalaron los jóvenes. De estos los dos principales fueron las peores condiciones de salud (64.8\%) y el de la expropiación de las tierras. Ambos señalamientos se identifican entre los primeros diez problemas de la Isla Municipio. Esto indica que para estos jóvenes existe una relación entre la presencia de la Marina en Vieques y los problemas de la Isla Municipio.

\footnotetext{
${ }^{1}$ Para esta premisa se podía seleccionar más de una alternativa.
} 
Los hallazgos de la investigación revelan que el 43 por ciento de los jóvenes encuestados indicaron que el problema de las prácticas de la Marina en Vieques sería resuelto: un 16.4 por ciento de los jóvenes señalaron que el issue no sería resuelto. La incertidumbre junto a la visión negativa de solución del problema manifiestan un proceso de reificación comparable a una falsa conciencia.

Estos hallazgos son cónsonos con el 91.4 por ciento de los jóvenes que indicaron sentirse con coraje y frustrados con los problemas de la Isla Municipio que afecta el nivel de participación en iniciativas para solucionar los problemas de Vieques con la Marina.

\section{Participación y organización}

Indica Paulo Freire que la transformación del mundo es realizada no dentro de la conciencia sino a través de la praxis. Así que, conciencia es más que percatarse de una situación excluyente, se trata de participar esperanzadoramente en el cambio de la misma (Barreto, Quiñónez, 2000). Estudios realizados con jóvenes han encontrado una pobre participación de este sector . Es por esto que es significativo que poco menos de la mitad de los jóvenes encuestados (43.3\%) participen de alguna actividad organizada para la solución de los problemas de Vieques. Enriquecendo a actividades y organizaciones, con su energía, valentía y disposición a transformar el mundo.

RAZONES PARA PARTICIPAR

\begin{tabular}{lcc}
\hline Motivo & Frecuencia $^{1}$ & Por ciento \\
\hline Cambiar la realidad de Vieques & 41 & 80.0 \\
En la unión esta la fuerza & 40 & 72.7 \\
Resultados del trabajo en grupo & 33 & 60.0 \\
Violaciones de derechos humanos & 21 & 38.1 \\
Sentido de justicia & 16 & 29.0 \\
Ideales & 15 & 27.2 \\
Participación de familiares & 8 & 14.5 \\
Ver al chico/a que le agrada & 6 & 10.9 \\
Distraerse & 5 & 9.0 \\
Políticos & 5 & 9.0 \\
Salir de la casa & 3 & 5.4 \\
Influencia de pares & 2 & 3.6 \\
Religiosos & 2 & 3.6 \\
Aceptación & 1 & 1.8 \\
\hline
\end{tabular}

${ }^{1}$ Para esta premisa se podía seleccionar más de una alternativa. 
Las razones principales para participar se encuentran relacionadas con los beneficios del trabajo en grupo para resolver los problemas existentes en la Isla de Vieques. Un 80 por ciento de los jóvenes señaló participar para cambiar la realidad de Vieques. Un 72.7 por ciento lo hacía porque en la unión está la fuerza entre otros motivos que evidencian que estos jóvenes favorecen y creen en el trabajo en equipo.

\section{Conclusiones}

Los jóvenes viequenses constituyen un grupo de jóvenes como cualquiera otro, son chicos y chicas que disfrutan de practicar algún deporte ir a la playa y ver televisión, con sus amigos. Por otro lado tienen preocupaciones y aspiraciones. Sin embargo, el haberse criado en un contexto de guerra, entre prácticas bélicas y una actividad civil en busca de condiciones dignas de vida junto a situaciones tales como el aislamiento geográfico de las principales instituciones de servicio, le han hecho desarrollar una visión de responsabilidad social diferente a otros jóvenes no expuestos a los elementos antes mencionados. Es tal vez por lo que ha sido su vida que en comparación con otros jóvenes puertorriqueños, los jóvenes viequenses entrevistados tienen un mayor nivel de participación social.

Sin duda, el dinamismo, la creatividad y la entrega de los jóvenes mantiene viva la llama de la esperanza por alcanzar un nuevo Vieques. Este es el espíritu por excelencia que sirve de sostén a las luchas sociales. Sin éste, la lucha por la justicia no tendría dónde descansar.

Por último uno de los participantes indica al respecto:

"Primero hay que luchar por lo que uno quiere de verdad, a pesar que uno tenga que caer, pero hay más satisfacción cuando se logra. Creo que la Marina se va de Vieques, se va porque se tiene que ir. Quizás me muera y no lo vea pero mi satisfacción será que mis hijos y mis nietos lo van a ver" 


\section{Bibliografía}

Ander-Egg, Ezequiel, (1995). Diccionario de Trabajo Social. Buenos Aires, Lumen.

Arteaga y Arteaga, (1999). Primer Estudio Generación. Hablemos.

Berger, Peter L., Luckmann, Thomas. (1979). La construcción social de la realidad. (5ta ed.) (Traducción por Silvia Zuleta). Buenos Aires: Amorrortu. (Trabajo original publicado en 1968).

Collazo, A. \& Rodríguez Roldán, N. (1993). Estudio de la Juventud Puertorriqueña. Universidad de Puerto Rico, Recinto de Río Piedras.

Departamento de Planificación. Vieques: Plan final de ordenamiento territorial. (2000, noviembre).

Departamento del Trabajo y Recursos Humanos de Puerto Rico (1997). Occupational Employment Statistics.

Enciclopedia Internacional de las Ciencias Sociales, Volumen 8 (1979). España: Crowell Collier \& Mc Millan. pp. 7-52.

Erickson, E. H. (1963). Childhood and Society (29th ed.). New York.

Follari, R., Hernández, J. \& Sánchez, F. (1984). Trabajo en común: Análisis y Perspectivas. Buenos Aires: Editorial Humanitas.

Fowlew, J. (1981). Stages of Farth: The pshycology of Human Development and the Quest for Meaning. San Francisco Harper Row.

Francia, A., Martín A., Salmeron, H. \& Esteban C. Tema I: Análisis de la Realidad. En: Análisis de la Realidad. Madrid: Editorial CCS, 1993. pp. 21-33.

Freire, Paulo (1974). Concientización. Argentina, Ediciones Búsqueda SAEIC. 
Grupo de Apoyo Técnico y Profesional. (2000, junio 6). Guías para el desarrollo sustentable en Vieques: Breves para la discusión.

Hopehayan, M. (1988). La participación y sus motivos. Acción Crítica, 24. pp.19- 30 .

Marx, K. (1970). A Contribution to the Critique of Political Economy. (Trabajo original en 1859). New York: International Publishers.

McCann-Erickson. (1995). Perfil Sicográfico del Joven Puertorriqueño entre las edades de 15-24 años. Puerto Rico: Ramallo Bros. Printing. Inc.

Resumen Ejecutivo de la Comisión Especial de Vieques. 1999, 5 de julio.

Rodríguez, V. (1997, febrero). Juventud Boricua en depresión. Diálogo, p. 3.

Vela, Andrés. (1990). Técnicas de concienciación. Indo American Press: Colombia.

Villarini, A. (1987). Principios para la integración del currículo. pp. 15-23. 